\title{
Telomere-independent functions of telomerase in nuclei, cytoplasm, and mitochondria
}

\author{
llaria Chiodi and Chiara Mondello* \\ Istituto di Genetica Molecolare, Consiglio Nazionale delle Ricerche, Pavia, Italy
}

\section{Edited by: \\ Claus M. Azzalin, Eidgenössische Technische Hochschule Zürich, \\ Switzerland \\ Susan M. Bailey, Colorado State \\ University, USA}

\section{Reviewed by:}

Xu-Dong Zhu, McMaster University, Canada

Yongmei Song, Chinese Academy of Medical Sciences and Peking Union Medical College, China

\section{${ }^{*}$ Correspondence:}

Chiara Mondello, Istituto di Genetica Molecolare, Consiglio Nazionale delle Ricerche, Via Abbiategrasso 207,

27100 Pavia, Italy.

e-mail:mondello@igm.cnr.it
Telomerase canonical activity at telomeres prevents telomere shortening, allowing chromosome stability and cellular proliferation. To perform this task, the catalytic subunit (telomerase reverse transcriptase, TERT) of the enzyme works as a reverse transcriptase together with the telomerase RNA component (TERC), adding telomeric repeats to DNA molecule ends. Growing evidence indicates that, besides the telomeric-DNA synthesis activity, TERT has additional functions in tumor development and is involved in many different biological processes, among which cellular proliferation, gene expression regulation, and mitochondrial functionality. TERT has been shown to act independently of TERC in the Wnt- $\beta$-catenin signaling pathway, regulating the expression of Wnt target genes, which play a role in development and tumorigenesis. Moreover, TERT RNA-dependent RNA polymerase activity has been found, leading to the genesis of double-stranded RNAs that act as precursor of silencing RNAs. In mitochondria, a TERT TERC-independent reverse transcriptase activity has been described that could play a role in the protection of mitochondrial integrity. In this review, we will discuss some of the extra-telomeric functions of telomerase.

\section{Keywords: telomerase, TERT, telomere, transformation, cancer, apoptosis, mitochondria, RNA interference}

\section{INTRODUCTION}

Telomerase has been discovered as the enzyme that maintains telomeric DNA, that is the DNA forming the physical ends of the eukaryotic chromosomes, the telomeres (Greider and Blackburn, 1985). In most eukaryotes, telomeric DNA is composed of tandem repetitions of short DNA sequences, in humans and in all vertebrates the TTAGGG hexanucleotide. In all organisms, telomeric DNA is bound to specific proteins (Blackburn and Gall, 1978; Moyzis et al., 1988; Palm and de Lange, 2008). Due to the catalytic properties of conventional DNA polymerases, telomeres require dedicated mechanisms for their replication and telomerase is the main telomere maintenance player.

Telomerase is an RNA-protein complex with reverse transcriptase activity, which contains both the protein catalytic subunit (telomerase reverse transcriptase, TERT) and the RNA template (telomerase RNA component, TERC) for the synthesis of the telomeric DNA (Collins, 2006). At each cell cycle, it binds to and elongates telomeres. When telomerase is low or absent, as in normal somatic cells, telomeres shorten at each cell division. Below a threshold length, telomeres activate DNA double strand break checkpoints, leading to cellular senescence, a permanent replicative arrest, or to cell death trough apoptosis (van Steensel etal., 1998; Masutomi etal., 2003; Rodier and Campisi, 2011). Telomere shortening can have a dramatic impact on stem cell proliferation, impairing their ability to regenerate tissues (Flores and Blasco, 2010). On the other hand, when telomerase is expressed constitutively, as in cancer cells, it allows telomere maintenance and an unlimited cellular proliferation (Shay and Bacchetti, 1997; Harley, 2008).

Because of its telomeric function, telomerase plays critical roles in cancer, aging, and degenerative diseases; nevertheless, accumulating evidence indicates that telomerase is also involved in these processes through functions independent of telomeric DNA synthesis. The purpose of this review is to discuss some of the recent discoveries about these telomere-independent functions.

\section{TERT, CANCER, AND APOPTOSIS}

Cloning of the TERT gene allowed demonstrating the role of telomere shortening in cellular senescence, as well as the role of telomerase in cellular immortalization (Bodnar et al., 1998). In fact, in several human somatic cells, exogenous TERT expression was found to be sufficient to reconstitute telomerase activity, stabilize telomere length, and consent an unlimited replicative potential. However, the study of genetically modified cells or mice in which TERT had been exogenously expressed also revealed novel telomerase functions in tumorigenesis (GonzalezSuarez etal., 2001; Artandi etal., 2002; Stewart etal., 2002; Canela et al., 2004).

Since many reviews have dealt with telomerase telomereindependent activities possibly involved in tumorigenesis (Mondello and Scovassi, 2004; Belgiovine et al., 2008; Bollmann, 2008; Cong and Shay, 2008; Majerska et al., 2011; Martinez and Blasco, 2011), here below we will only briefly remind a few relevant points concerning this topic.

1. TERT expression can promote cell growth and proliferation independently of telomere elongation by inducing or inhibiting the expression of pro-proliferative and anti-proliferative genes, respectively. By that, it enables the cells to proliferate in the absence of mitogenic stimuli, a hallmark of cancer cells Geserick et al., 2006). (Stampfer et al., 2001; Lindvall et al., 2003; Smith et al., 2003; 
2. TERT can increase resistance to chemotherapeutic agents and pro-apoptotic stimuli, possibly blocking the mitochondrial death pathway (Dudognon et al., 2004; Del Bufalo et al., 2005; Mondello et al., 2006; Lee et al., 2008).

3. TERT can modulate chromatin structure and response to DNA damage (Sharma et al., 2003; Masutomi et al., 2005; Gu et al., 2008).

4. TERT can increase cancer cell fitness improving mitochondrial activity and resistance to apoptosis (see Section "TERCindependent Reverse Transcriptase Activity" and references therein).

5. TERT can stabilize telomeres in a telomere-capping dependent manner increasing cell's lifespan without telomere lengthening (Zhu et al., 1999; Kim et al., 2003; Mukherjee et al., 2011).

Recently, Okamoto et al. (2011) suggested TERT involvement in carcinogenesis through cancer stem cell (CSC) maintenance. According to these authors, TERT forms a complex with a transcriptional regulator, BRG1 (see below), and a GTP-binding protein overexpressed in stem cells and cancers, nucleostemin (Tsai and McKay, 2002), which is essential to drive transcriptional programs relevant for the maintenance of the CSC phenotype (Okamoto et al., 2011). This TERT function is independent of its role at telomeres and could contribute to tumorigenesis by increasing the proportion of CSCs within a tumor.

\section{TERT AND GENE EXPRESSION REGULATION}

Telomere shortening due to insufficient telomerase activity undoubtedly threaten organisms' health, as shown in experimental mouse models and in human syndromes, such as for example Dyskeratosis congenita (Dokal, 2011). In this syndrome, mutations in genes coding for TERT, TERC, or dyskerin, another subunit of human telomerase (Cohen et al., 2007), lead to telomere shortening and organ failure, probably because of a reduction in stem cell compartments (Flores and Blasco, 2010). Nevertheless, evidence has been reported that TERT can also operate in cell physiology through additional mechanisms.

Studies on mice overexpressing TERT in the skin suggested that TERT is involved in stem cell mobilization (Flores et al., 2005; Sarin et al., 2005). These works demonstrated that TERT overexpression promotes activation of quiescent bulge stem cells and hair growth, independently of telomere elongation, indicating that TERT can activate pathways involved in stem cell renewal. To this regard, Choi et al. (2008) showed that conditionally TERT expression in mouse skin induces a gene expression profile resembling the transcriptional program regulated by Wnt, a well known player in stem cell maintenance and proliferation, as well as in cellular transformation (Van Mater et al., 2003; Reya and Clevers, 2005; Wege et al., 2011). Park et al. (2009) undisclosed the connection between TERT, stem cell proliferation and the Wnt pathway showing that TERT directly modulates the Wnt pathway by acting as a transcription factor in $\beta$-catenin complexes. In fact, TERT interacts with BRG1, a chromatin remodeler binding to $\beta$-catenin and involved in the Wnt signaling (Barker et al., 2001), and promotes the expression of several $\beta$-catenin target genes in a BRG1dependent way. Moreover, TERT was found not only to bind to promoters responsive to Wnt signaling, but also to interact with the same promoter elements recognized by BRG1 and $\beta$-catenin (Park et al., 2009).

Recently, the telomere-independent TERT role in Wnt signaling was shown to be involved in kidney pathologies, such as collapsing glomerulopathies (Shkreli et al., 2012). Both idiopathic and HIV-associated collapsing glomerulopathies are characterized by proliferation of glomerular differentiated epithelial cells, the podocytes. Podocytes are an essential part of the glomerular filtration membrane and their proliferation compromises the kidney filtration barrier, leading to severe organ damage (Barisoni et al., 2000). Using a transgenic mouse model conditionally expressing TERT, Shkreli et al. (2012) showed that induction of the transgene led to TERT expression in several tissues and to the development of kidney abnormalities resembling those observed in human collapsing glomerulopathies, including podocyte proliferation. TERT effects on glomerulus were independent of its catalytic activity, but linked to its Wnt signaling induction, with increased expression and nuclear localization of $\beta$-catenin. More importantly, TERT upregulation and $\beta$-catenin stabilization were also found in human and mouse HIV-associated glomerulopathies, indicating that TERT-mediated Wnt activation can be required for podocyte proliferation in these diseases. The observation that TERT silencing and Wnt pathway inactivation in transgenic mice can revert the podocyte phenotype suggests TERT and Wnt as new targets to fight collapsing glomerulopathies.

Bernardes de Jesus et al. (2012) found indications for Wnt pathway activation in several organs, including kidney, of 2-year-old mice treated with an adeno-associated virus (AAV) expressing mouse TERT. However, in contrast with Shkreli et al.'s (2012) results, these authors did not report any signs of kidney failure in these mice, despite increased expression of active $\beta$-catenin and of its target gene cyclin D1 in this organ. In these mice, TERT had an anti-aging effect strictly dependent on its catalytic telomere lengthening activity, leading to an extension of mouse life span, with beneficial effects on health and fitness (Bernardes de Jesus et al., 2012). A few factors could explain the differences in the two groups' finding, including the lack of a complete Wnt pathway activation in the mouse studied by Bernardes de Jesus et al. (2012), the different mouse age at the time of telomerase expression induction (3 weeks vs. 2 years), possible difference in TERT levels, or the different mouse strains used for the experiments (FVB/N strain vs. C57BL6). However, Shkreli et al.'s (2012) observation of TERT upregulation in human HIV-associated nephropathy, in the absence of any experimental manipulation, suggests that TERT overexpression could be a threat for the organism and further experiments should be performed to ensure a safe use of TERT exogenous expression for medical purposes.

Recent reports have shown that the relationship between TERT and Wnt pathway activation is in fact bidirectional, both in embryonic stem (ES) cells and cancer (Hoffmeyer et al., 2012; Zhang et al., 2012). Hoffmeyer et al. (2012) found that $\beta$-catenin deficient mice ES cells had shorter telomeres and lower telomerase activity compared with wild type mice, while longer telomeres and higher telomerase levels were detected in ES cells expressing an activated $\beta$-catenin. A similar effect on telomere length and telomerase activity has been shown by inducing or repressing $\beta$-catenin expression in human cancer cell lines (Zhang et al., 2012). It seems that 
$\beta$-catenin regulates TERT expression in ES cells through the binding to the pluripotent transcription factor Klf4, while in human cancer cells, TERT appears as a direct target of $\beta$-catenin/TCF4mediated transcription. Thus, activation of the Wnt pathway during transformation could also participate in some carcinogenesis processes through telomerase activity induction and telomere stabilization.

Although the exogenous TERT expression experiments clearly indicate telomerase functions independent of its telomere lengthening activity, comparison of $\mathrm{TERC}^{-/-}$mice with $\mathrm{TERT}^{-/-}$ animals did not show any differences in their phenotypes that could imply TERT extra-telomeric activities (Vidal-Cardenas and Greider, 2010; Strong et al., 2011). Even if it is not possible to exclude that overexpression experiments lead to phenotypes not related to the real function of the gene under investigation, it is also conceivable that animals with TERT ablation since the initial phases of embryogenesis could develop pathways compensating for TERT inactivation.

\section{TERC-INDEPENDENT TERT CATALYTIC ACTIVITIES}

Historically, TERT has been described as an RNA-dependent DNA polymerase in association with TERC, whose catalytic activity is carried out at telomeres (Greider and Blackburn, 1985). More recently, a novel TERT function has been found in post-transcriptional gene silencing as an RNA-dependent RNA polymerase (RdRP) paired to a mitochondrial non-coding RNA (Maida et al., 2009); TERT is closely related to viral RdRPs and, currently, it is the only RdRP identified in mammals (Maida and Masutomi, 2011). Moreover, in mitochondria, TERT practices a TERC-independent reverse transcriptase function that uses mttRNAs as template (Sharma et al., 2012). Finally, the eclectic nature of TERT catalytic subunit was confirmed by Lue et al. (2005), who demonstrated that, in particular metal ion concentration, TERT can work as terminal transferase in a template- and RNAindependent way (Lue et al., 2005). Mutations in TERT catalytic domain impair all these enzymatic activities, despite their remarkable differences in template usage and final products, and hence may affect different physiological processes simultaneously.

\section{TERC-INDEPENDENT RNA-DEPENDENT RNA POLYMERASE ACTIVITY}

Isolation of TERT-RNA complexes from HeLa cells overexpressing hTERT demonstrated the existence of at least two equivalent RNA partners, TERC and mitochondrial RNA processing endoribonuclease (RMRP; Maida et al., 2009), a non-coding RNA whose mutations cause the inherited pleiotropic syndrome Cartilagehair hypoplasia (Ridanpaa et al., 2001). TERT-RMRP complex exhibited RdRP activity and led to production of double-stranded RMRP RNA molecules, subsequently processed into 22-nucleotide siRNAs by RNA-induced silencing complex (RISC). These siRNAs suppressed RMRP expression, implying TERT role in controlling gene expression through RNA interference (Maida et al., 2009). Interestingly, mutations in TERT catalytic domain impaired both the DNA and RNA polymerase activity.

TERT RdRP has been recently found to have a role in TERT control of cellular proliferation (Smith et al., 2003; Mukherjee et al., 2011). In human mammary epithelial cells (HMECs), TERT transduction enhanced cellular proliferation, both increasing cell division and reducing apoptosis (Mukherjee et al., 2011). This effect on cellular proliferation was associated with alterations in cell cycle regulator proteins, such as cyclin D1 and A2, E2F and $\mathrm{pRB}$, required telomerase catalytic activity, but was independent of TERT activation of Wnt signaling (Mukherjee et al., 2011). In fact, no transcription induction of Wnt-responsive genes, targets of TERT (Choi et al., 2008), was observed in TERTtransduced HMECs. Interestingly, Mukherjee et al. (2011) linked TERT enhancement of cellular proliferation to a decrease in RMPR levels. Exogenous TERT expression led to a remarkable reduction of RMRP levels in HMECs, as a result of a DICER-dependent processing of double-stranded RMRP molecules synthesized by RMRP-TERT complex itself. Notably, RMRP shRNA knockdown gave results comparable to TERT exogenous expression, both in lowering RMRP levels and improving cellular proliferation. On the other hand, RMRP knockdown in TERT expressing cells triggered only a minor additional decrease in RMRP levels and a slight increase in proliferation rate. These data suggest a novel role of TERT as modulator of cellular proliferation mediated by small interfering RNAs derived from RNA-dependent DNA polymerase activity of TERT (Mukherjee et al., 2011).

\section{TERC-INDEPENDENT REVERSE TRANSCRIPTASE ACTIVITY}

As mentioned in the second paragraph, TERT is a dually localized protein. It is mainly present in the nucleus, but about $10-20 \%$ of cellular TERT localizes in mitochondria $(\mathrm{mt})$, both in telomerase-expressing normal cells and in cancer cells. TERT has an N-terminal mitochondrial targeting signal (Santos et al., 2004; Sharma et al., 2012) and is imported into the organelle, probably through the binding to a complex containing translocases of the outer and inner membranes. TERT also binds to several mtDNA regions (Haendeler et al., 2009; Sharma et al., 2012). Under oxidative stress, TERT is reversibly excluded from the nucleus (Haendeler et al., 2003, 2004; Santos et al., 2004,2006) and localizes in mitochondria (Ahmed et al., 2008). Several reports have shown that mt-TERT improves mitochondrial function and stress resistance, independently of its telomeric function. This non-telomeric TERT activity could contribute to tumorigenesis by increasing tumor cell survival (Ahmed et al., 2008; Haendeler et al., 2009; Indran et al., 2011; for reviews on telomerase and mitochondria see Saretzki, 2009; Gordon and Santos, 2010; Indran et al., 2010).

Telomerase reverse transcriptase catalytic activity seems to be required for TERT mitochondrial effects (Santos et al., 2006). However, recent evidence indicates that TERC is not present in mitochondria, suggesting that TERT reverse transcriptase activity is independent of TERC (Sharma et al., 2012). In support to this hypothesis, Sharma et al. (2012) showed that wild type TERT performed its mitochondrial functions even when expressed in human VA13 cells, which use the alternative lengthening of telomeres (ALT) mechanism and does not contain TERC. Moreover, in the same cells, a dominant negative form of the enzyme was inactive.

Given that in mitochondria TERT binds to several mt-tRNAs and many reverse transcriptases use cellular tRNAs to perform their synthesis (Herschhorn and Hizi, 2010), mt-TERT could use tRNAs rather than TERC for cDNA synthesis. To test this hypothesis, Sharma et al. (2012) first translated TERT in rabbit 
reticulocyte lysates (RRLs) in the presence of TERC and demonstrated that TERT was able to synthesize telomeric repeats in a TRAP based assay. Subsequently, they added total cellular RNAs from TERC positive HeLa cells or TERC negative VA13 cells to the TERT-RRL reaction mixture, as well as random hexamers to prime the reactions. Analysis for the presence of cDNAs was then performed through PCR with primers for different mt-tRNA genes. In these in vitro reactions, TERT could clearly perform cDNA synthesis in the absence of TERC. Preincubation with TERC abolished the synthesis of non-telomeric cDNAs, while incubation with RNAs from TERC-positive HeLa cells reduced the reverse transcription of mt-tRNAs, suggesting that TERC competes with the other RNAs for TERT binding. In the absence of TERT, no products were observed. To confirm these data, Sharma et al. (2012) performed an in vitro reverse transcriptase reaction using in vitro transcribed mt-tRNAcys as template together with wtTERT or a dominantnegative mutant form of the enzyme and found the cDNA corresponding to the template in the first case, but not in the second one. Thus, this work strongly indicates that mt-TERT can work as a TERC-independent reverse transcriptase that uses mt-tRNAs as template.

So far, the function of this novel TERT activity is unknown. However, Sharma et al. (2012) suggested its role in mt-DNA replication and/or repair. Since a reverse transcriptase activity could be involved in the priming of the light mt-chain origin (Kaguni, 2004), TERT might have a part in this DNA replication step. Moreover, TERT's function in mt-DNA replication might partially explain its ability to counteract mt-DNA loss after ethidium bromide exposure in telomerase deficient mice (Haendeler et al., 2009). Very little is known about DNA repair in mitochondria (Liu and Demple, 2010). In yeast, evidence has been reported that RNA could be used to repair DNA double strand breaks and then converted into DNA by a reverse transcriptase activity (Storici et al., 2007). It is possible that TERT participate in this process also in mammalian cells.

In addition to TERT playing a protective role in mitochondria, a recent report demonstrated the influence of telomere length on the control of mitochondrial fitness (Sahin et al., 2011). This report showed that telomere dysfunction in TERT and TERC late generation knockout mice led to a p53-mediated repression of the master regulators of mitochondrial physiology and metabolism peroxisome proliferator-activated receptor gamma, coactivator-1 alpha and beta (PGC- $1 \alpha$ and PGC-1 $\beta$ ), and to a repression of the downstream pathway. This resulted in altered mitochondrial biogenesis and function, decreased gluconeogenesis, cardiomyopathy, and increased reactive oxygen species. Restoring TERT or PGC- $1 \alpha$ expression in the TERT knockout mice, or deleting TP53 in the germline, reverted the phenotype.

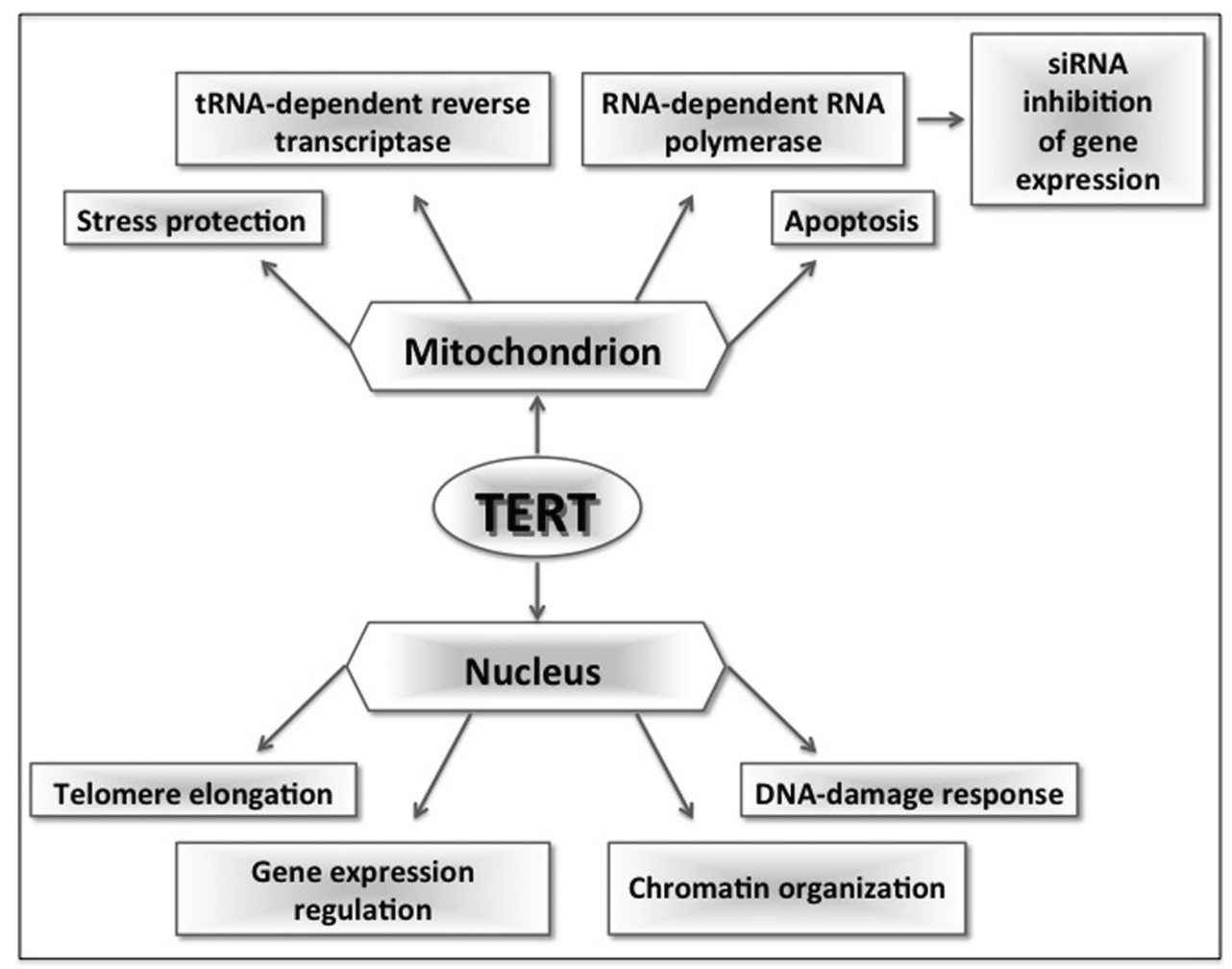

FIGURE 1 | Schematic representation of multiple TERT functions. In the nucleus, TERT can elongate telomeres binding to TERC; binding to BRG1, it can work as a coactivator of the Wnt pathway and regulate gene expression; finally, silencing experiments in somatic cells showed that TERT is involved in chromatin organization and DNA-damage response. In mitochondria, TERT plays a role in stress response, apoptosis, and in the maintenance of mitochondrial fitness; moreover, it can interact with RNAs other than TERC acquiring an additional RNA transcriptase activity or an RNA-dependent RNA polymerase activity. This last activity seems to lead the production of siRNAs that are involved in post-transcriptional gene expression regulation. 


\section{CONCLUSION AND FUTURE PERSPECTIVES}

A large body of evidence indicates that the telomerase complex, and in particular its TERT subunit, plays several functions beyond the maintenance of telomere length and chromosome stability (Figure 1). These functions can impact normal cell physiology but also cancer cells, giving them a selective advantage. As far as cancer cells are concerned, the discovery of pathways in which telomerase can intervene and promote tumorigenesis beyond telomeres could highlight new possible targets for tumor therapy. Little is known on mitochondrial TERT in normal somatic cells and on its new role as mitochondrial RNA-dependent DNA polymerase. Further

\section{REFERENCES}

Ahmed, S., Passos, J. F., Birket, M. J., Beckmann, T., Brings, S., Peters, H., et al. (2008). Telomerase does not counteract telomere shortening but protects mitochondrial function under oxidative stress. J. Cell Sci. 121, 1046-1053.

Artandi, S. E., Alson, S., Tietze, M. K., Sharpless, N. E., Ye, S., Greenberg, R. A., et al. (2002). Constitutive telomerase expression promotes mammary carcinomas in aging mice. Proc. Natl. Acad. Sci. U.S.A. 99, 8191-8196.

Barisoni, L., Mokrzycki, M., Sablay, L., Nagata, M., Yamase, H., and Mundel, P. (2000). Podocyte cell cycle regulation and proliferation in collapsing glomerulopathies. Kidney Int. 58, 137-143.

Barker, N., Hurlstone, A., Musisi, H., Miles, A., Bienz, M., and Clevers, H. (2001). The chromatin remodelling factor Brg-1 interacts with beta-catenin to promote target gene activation. EMBO J. 20, 4935-4943.

Belgiovine, C., Chiodi, I., and Mondello, C. (2008). Telomerase: cellular immortalization and neoplastic transformation. Multiple functions of a multifaceted complex. Cytogenet. Genome Res. 122, 255-262.

Bernardes de Jesus, B., Vera, E., Schneeberger, K., Tejera, A. M., Ayuso, E., Bosch, F., et al. (2012). Telomerase gene therapy in adult and old mice delays aging and increases longevity without increasing cancer. $E M B O$ Mol. Med. 4, 691-704

Blackburn, E. H., and Gall, J. G. (1978), A tandemly repeated sequence at the termini of the extrachromosomal ribosomal RNA genes in Tetrahymena. J. Mol. Biol. 120, 33-53.

Bodnar, A. G., Ouellette, M., Frolkis, M., Holt, S. E., Chiu, C. P., Morin, G. B., et al. (1998). Extension of lifespan by introduction of telomerase into normal human cells. Science 279 , 349-352.

Bollmann, F. M. (2008). The many faces of telomerase: emerging extratelomeric effects. Bioessays 30, 728-732.
Canela, A., Martin-Caballero, J., Flores, J. M., and Blasco, M. A. (2004). Constitutive expression of tert in thymocytes leads to increased incidence and dissemination of T-cell lymphoma in Lck-Tert mice. Mol. Cell Biol. 24, 4275-4293.

Choi, J., Southworth, L. K., Sarin, K. Y., Venteicher, A. S., Ma, W., Chang, W., et al. (2008). TERT promotes epithelial proliferation through transcriptional control of a Myc- and Wnt-related developmental program. PLoS Genet. 4, e10. doi: 10.1371/journal.pgen.0040010

Cohen, S. B., Graham, M. E., Lovrecz, G. O., Bache, N., Robinson, P. J., and Reddel, R. R. (2007). Protein composition of catalytically active human telomerase from immortal cells. Science 315, 1850-1853.

Collins, K. (2006). The biogenesis and regulation of telomerase holoenzymes. Nat. Rev. Mol. Cell Biol. 7, 484-494.

Cong, Y., and Shay, J. W. (2008). Actions of human telomerase beyond telomeres. Cell Res. 18, 725-732.

Del Bufalo, D., Rizzo, A., Trisciuoglio, D., Cardinali, G., Torrisi, M R., Zangemeister-Wittke, U., et al. (2005). Involvement of hTERT in apoptosis induced by interference with Bcl-2 expression and function. Cell Death Differ. 12, 1429-1438.

Dokal, I. (2011). Dyskeratosis congenita. Hematology Am. Soc. Hematol. Educ. Program 2011, 480-486.

Dudognon, C., Pendino, F., Hillion, J., Saumet, A., Lanotte, M., and SegalBendirdjian, E. (2004). Death receptor signaling regulatory function for telomerase: hTERT abolishes TRAILinduced apoptosis, independently of telomere maintenance. Oncogene 23, 7469-7474.

Flores, I., Cayuela, M. L., and Blasco, M. A. (2005). Effects of telomerase and telomere length on epidermal stem cell behavior. Science 309, 1253-1256.

Flores, I., and Blasco, M. A. (2010). The role of telomeres and telomerase in stem cell aging. FEBS Lett. 584, 3826-3830.

investigation of these topics might help clarify TERT role on aging, in which dysfunctional mitochondria seem to play a relevant role. Particularly important will be to gain the tools, so far limited, to study TERT functions in cellular physiological conditions and, especially, in normal somatic cells, in which the low abundance of the protein has limited its analysis.

\section{ACKNOWLEDGMENTS}

Work in Chiara Mondello's laboratory is supported by Fondazione Cariplo (grant no. 2011-0370). We thank Dr. Aya Biran for help in editing the English style.

Geserick, C., Tejera, A., GonzalezSuarez, E., Klatt, P., and Blasco, M. A. (2006). Expression of mTert in primary murine cells links the growthpromoting effects of telomerase to transforming growth factor-beta signaling. Oncogene 25, 4310-4319.

Gonzalez-Suarez, E., Samper, E., Ramirez, A., Flores, J. M., MartinCaballero, J., Jorcano, J. L., et al. (2001). Increased epidermal tumors and increased skin wound healing in transgenic mice overexpressing the catalytic subunit of telomerase, mTERT, in basal keratinocytes. EMBO J. 20, 2619-2630.

Gordon, D. M., and Santos, J. H. (2010). The emerging role of telomerase reverse transcriptase in mitochondrial DNA metabolism. J. Nucleic Acids 2010, 390791.

Greider, C. W., and Blackburn, E. H. (1985). Identification of a specific telomere terminal transferase activity in Tetrahymena extracts. Cell 43, 405-413.

$\mathrm{Gu}$, B. W., Bessler, M., and Mason, P. J. (2008). A pathogenic dyskerin mutation impairs proliferation and activates a DNA damage response independent of telomere length in mice. Proc. Natl. Acad. Sci. U.S.A. 105, 10173-10178.

Haendeler, J., Hoffmann, J., Rahman, S., Zeiher, A. M., and Dimmeler, S. (2003). Regulation of telomerase activity and anti-apoptotic function by protein-protein interaction and phosphorylation. FEBS Lett. 536, 180-186.

Haendeler, J., Hoffmann, J., Zeiher, A. M., and Dimmeler, S. (2004). Antioxidant effects of statins via S-nitrosylation and activation of thioredoxin in endothelial cells: a novel vasculoprotective function of statins. Circulation 110, 856-861.

Haendeler, J., Drose, S., Buchner, N., Jakob, S., Altschmied, J., Goy, C. et al. (2009). Mitochondrial telomerase reverse transcriptase binds to and protects mitochondrial DNA and function from damage. Arterioscler. Thromb. Vasc. Biol. 29, 929-935.
Harley, C. B. (2008). Telomerase and cancer therapeutics. Nat. Rev. Cancer 8, 167-179.

Herschhorn, A., and Hizi, A. (2010). Retroviral reverse transcriptases. Cell Mol. Life Sci. 67, 2717-2747.

Hoffmeyer, K., Raggioli, A., Rudloff, S., Anton, R., Hierholzer, A., Del Valle, I., et al. (2012). Wnt/beta-catenin signaling regulates telomerase in stem cells and cancer cells. Science 336, 1549-1554.

Indran, I. R., Hande, M. P., and Pervaiz, S. (2010). Tumor cell redox state and mitochondria at the center of the non-canonical activity of telomerase reverse transcriptase. Mol. Aspects Med. 31, 21-28.

Indran, I. R., Hande, M. P., and Pervaiz, S. (2011). hTERT overexpression alleviates intracellular ROS production, improves mitochondrial function, and inhibits ROS-mediated apoptosis in cancer cells. Cancer Res. 71, 266-276.

Kaguni, L. S. (2004). DNA polymerase gamma, the mitochondrial replicase. Annu. Rev. Biochem. 73, 293-320.

Kim, M., Xu, L., and Blackburn, E. H. (2003). Catalytically active human telomerase mutants with allele-specific biological properties. Exp. Cell Res. 288, 277-287.

Lee, J., Sung, Y. H., Cheong, C., Choi, Y. S., Jeon, H. K., Sun, W., et al. (2008). TERT promotes cellular and organismal survival independently of telomerase activity. Oncogene 27, 3754-3760.

Lindvall, C., Hou, M., Komurasaki, T., Zheng, C., Henriksson, M., Sedivy, J. M., et al. (2003). Molecular characterization of human telomerase reverse transcriptase-immortalized human fibroblasts by gene expression profiling: activation of the epiregulin gene. Cancer Res. 63, 1743-1747.

Liu, P., and Demple, B. (2010). DNA repair in mammalian mitochondria: much more than we thought? Environ. Mol. Mutagen. 51, 417-426.

Lue, N. F., Bosoy, D., Moriarty, T. J., Autexier, C., Altman, B., and Leng, S. (2005). Telomerase can act as a 
template- and RNA-independent terminal transferase. Proc. Natl. Acad. Sci. U.S.A. 102, 9778-9783.

Maida, Y., Yasukawa, M., Furuuchi, M., Lassmann, T., Possemato, R., Okamoto, N., et al. (2009). An RNAdependent RNA polymerase formed by TERT and the RMRP RNA. Nature 461, 230-235.

Maida, Y., and Masutomi, K. (2011). RNA-dependent RNA polymerases in RNA silencing. Biol. Chem. 392, 299-304.

Majerska, J., Sykorova, E., and Fajkus, J. (2011). Non-telomeric activities of telomerase. Mol. Biosyst. 7, 1013-1023.

Martinez, P., and Blasco, M. A. (2011). Telomeric and extra-telomeric roles for telomerase and the telomerebinding proteins. Nat. Rev. Cancer 11, 161-176.

Masutomi, K., Yu, E. Y., Khurts, S., BenPorath, I., Currier, J. L., Metz, G. B. et al. (2003). Telomerase maintains telomere structure in normal human cells. Cell 114, 241-253.

Masutomi, K., Possemato, R., Wong, J. M., Currier, J. L., Tothova, Z., Manola, J. B., etal. (2005). The telomerase reverse transcriptase regulates chromatin state and DNA damage responses. Proc. Natl. Acad. Sci. U.S.A. 102, 8222-8227.

Mondello, C., and Scovassi, A. I. (2004). Telomeres, telomerase, and apoptosis. Biochem. Cell Biol. 82, 498-507.

Mondello, C., Bottone, M. G., Noriki, S., Soldani, C., Pellicciari, C., and Scovassi, A. I. (2006). Oxidative stress response in telomerase-immortalized fibroblasts from a centenarian. Ann. N. Y. Acad. Sci. 1091, 94-101.

Moyzis, R. K., Buckingham, J. M., Cram, L. S., Dani, M., Deaven, L. L., Jones, M. D., et al. (1988). A highly conserved repetitive DNA sequence, (TTAGGG)n, present at the telomeres of human chromosomes. Proc. Natl. Acad. Sci. U.S.A. 85, 6622-6626.

Mukherjee, S., Firpo, E. J., Wang, Y., and Roberts, J. M. (2011). Separation of telomerase functions by reverse genetics. Proc. Natl. Acad. Sci. U.S.A. 108, E1363-E1371.

Okamoto, N., Yasukawa, M., Nguyen, C., Kasim, V., Maida, Y., Possemato, R., et al. (2011). Maintenance of tumor initiating cells of defined genetic composition by nucleostemin. Proc. Natl. Acad. Sci. U.S.A. 108, 20388-20393.

Palm, W., and de Lange, T. (2008). How shelterin protects mammalian telomeres. Annu. Rev. Genet. 42, 301-334.

Park, J. I., Venteicher, A. S., Hong, J. Y., Choi, J., Jun, S., Shkreli, M., et al. (2009). Telomerase modulates Wnt signalling by association with target gene chromatin. Nature 460, 66-72.

Reya, T., and Clevers, H. (2005). Wnt signalling in stem cells and cancer. Nature 434, 843-850.

Ridanpaa, M., van Eenennaam, H., Pelin, K., Chadwick, R., Johnson, C., Yuan, B., et al. (2001). Mutations in the RNA component of RNase MRP cause a pleiotropic human disease, cartilage-hair hypoplasia. Cell 104, 195-203.

Rodier, F., and Campisi, J. (2011). Four faces of cellular senescence. J. Cell Biol. 192, 547-556.

Sahin, E., Colla, S., Liesa, M., Moslehi, J., Muller, F. L., Guo, M., et al. (2011). Telomere dysfunction induces metabolic and mitochondrial compromise. Nature 470, 359-365.

Santos, J. H., Meyer, J. N., Skorvaga, M., Annab, L. A., and Van Houten, B. (2004). Mitochondrial hTERT exacerbates free-radicalmediated mtDNA damage. Aging Cell 3, 399-411.

Santos, J. H., Meyer, J. N., and Van Houten, B. (2006). Mitochondrial localization of telomerase as a determinant for hydrogen peroxideinduced mitochondrial DNA damage and apoptosis. Hum. Mol. Genet. 15, 1757-1768.

Saretzki, G. (2009). Telomerase, mitochondria and oxidative stress. Exp. Gerontol. 44, 485-492.

Sarin, K. Y., Cheung, P., Gilison, D., Lee, E., Tennen, R. I., Wang, E., et al. (2005). Conditional telomerase induction causes proliferation of hair follicle stem cells. Nature 436, 1048-1052.

Sharma, G. G., Gupta, A., Wang, H., Scherthan, H., Dhar, S., Gandhi, V., et al. (2003). hTERT associates with human telomeres and enhances genomic stability and DNA repair. Oncogene 22, 131-146.

Sharma, N. K., Reyes, A., Green, P., Caron, M. J., Bonini, M. G., Gordon, D. M., et al. (2012). Human telomerase acts as a hTR-independent reverse transcriptase in mitochondria. Nucleic Acids Res. 40, 712-725.

Shay, J. W., and Bacchetti, S. (1997). A survey of telomerase activity in human cancer. Eur. J. Cancer 33, 787-791.

Shkreli, M., Sarin, K. Y., Pech, M. F., Papeta, N., Chang, W., Brockman, S. A., et al. (2012). Reversible cellcycle entry in adult kidney podocytes through regulated control of telomerase and Wnt signaling. Nat. Med. 18, 111-119.

Smith, L. L., Coller, H. A., and Roberts, J. M. (2003). Telomerase modulates expression of growthcontrolling genes and enhances cell proliferation. Nat. Cell Biol. 5, 474-479.

Stampfer, M. R., Garbe, J., Levine, G. Lichtsteiner, S., Vasserot, A. P., and Yaswen, P. (2001). Expression of the telomerase catalytic subunit, hTERT, induces resistance to transforming growth factor beta growth inhibition in pl6INK4A(-) human mammary epithelial cells. Proc. Natl. Acad. Sci. U.S.A. 98, 4498-4503.

Stewart, S. A., Hahn, W. C., O'Connor B. F., Banner, E. N., Lundberg, A. S. Modha, P., et al. (2002). Telomerase contributes to tumorigenesis by a telomere length-independent mechanism. Proc. Natl. Acad. Sci. U.S.A 99, 12606-12611.

Storici, F., Bebenek, K., Kunkel, T. A., Gordenin, D. A., and Resnick, M. A. (2007). RNA-templated DNA repair. Nature 447, 338-341.

Strong, M. A., Vidal-Cardenas, S. L., Karim, B., Yu, H., Guo, N., and Greider, C. W. (2011). Phenotypes in mTERT $^{+/-}$and mTERT $^{-/-}$mice are due to short telomeres, not telomere-independent functions of telomerase reverse transcriptase. $\mathrm{Mol}$. Cell Biol. 31, 2369-2379.

Tsai, R. Y., and McKay, R. D. (2002). A nucleolar mechanism controlling cell proliferation in stem cells and cancer cells. Genes Dev. 16, 2991-3003.

Van Mater, D., Kolligs, F. T., Dlugosz, A. A., and Fearon, E. R. (2003). Transient activation of beta-catenin signaling in cutaneous keratinocytes is sufficient to trigger the active growth phase of the hair cycle in mice. Genes Dev. 17, 1219-1224.

van Steensel, B., Smogorzewska, A., and De Lange, T. (1998). TRF2 protects human telomeres from end-to-end fusions. Cell 92, 401-413.

Vidal-Cardenas, S. L., and Greider, C. W. (2010). Comparing effects of mTR and mTERT deletion on gene expression and DNA damage response: a critical examination of telomere length maintenance-independent roles of telomerase. Nucleic Acids Res. $38,60-71$.

Wege, H., Heim, D., Lütgehetmann, M., Dierlamm, J., Lohse, A. W., and Brümmendorf, T. H. (2011). Forced activation of $\beta$-catenin signaling supports the transformation of hTERT-immortalized human fetal hepatocytes. Mol. Cancer Res. 9, 1222-1231.

Zhang, Y., Toh, L. L., Lau, P., and Wang, P. (2012). Telomerase reverse transcriptase (TERT) is a novel target of $\mathrm{Wnt} / \beta$-catenin pathway in human cancer. J. Biol. Chem. doi: 10.1074/jbc.M112.368282 [Epub ahead of print].

Zhu, J., Wang, H., Bishop, J. M., and Blackburn, E. H. (1999). Telomerase extends the lifespan of virus-transformed human cells without net telomere lengthening. Proc. Natl. Acad. Sci. U.S.A. 96, 3723-3728.

Conflict of Interest Statement: The authors declare that the research was conducted in the absence of any commercial or financial relationships that could be construed as a potential conflict of interest.

Received: 31 July 2012; accepted: 18 September 2012; published online: 28 September 2012.

Citation: Chiodi I and Mondello C (2012) Telomere-independent functions of telomerase in nuclei, cytoplasm, and mitochondria. Front. Oncol. 2:133. doi: 10.3389/fonc. 2012.00133

This article was submitted to Frontiers in Cancer Molecular Targets and Therapeutics, a specialty of Frontiers in Oncology. Copyright (C) 2012 Chiodi and Mondello. This is an open-access article distributed under the terms of the Creative Commons Attribution License, which permits use, distribution and reproduction in other forums, provided the original authors and source are credited and subject to any copyright notices concerning any thirdparty graphics etc. 